\title{
Models of optimization of combination of production of agrarian products and harvesting of wild food resources
}

\author{
Yaroslav Ivanyo $^{1}$, Sofia Petrova $^{1,}{ }^{*}$, Margarita Barsukova $^{1}$, and Gantulga Gombo ${ }^{2}$ \\ ${ }^{1}$ Irkutsk State Agricultural University named after A.A. Ezhevsky Molodezhny, Irkutsk, Russia \\ ${ }^{2}$ Mongolian University of Life Sciences, Ulaanbaatar, Mongolia
}

\begin{abstract}
The article is devoted to the results of modeling the combination of agricultural production and harvesting of wild food resources. Agricultural enterprise models that are able to expand food production activities through the use of wild plants are proposed. A prerequisite for the application of the developed linear programming models with uncertain parameters is the availability of sufficient reserves of wild food resources located at relatively small distances from the farm. This condition is acceptable for many farms in the Irkutsk region. As optimization problems, linear parametric problems with interval and random estimates are used. With sustainable agriculture, the uncertain values are yield, labor costs and prices for harvesting and selling wild plants. The models used to manage the activities of agricultural producers of the Irkutsk region showed additional opportunities for the development of the regional agro-industrial complex.
\end{abstract}

\section{Introduction}

In regions with rich wild food products [1-3] and developed agricultural production, which include the Irkutsk region, it is urgent to solve problems associated with the activities of an agricultural enterprise for receiving of agricultural and wild food products. In the region, the subprogram "Development of the sphere of harvesting, processing and marketing of food forest resources and medicinal plants in the Irkutsk region" for 2019 2024 was adopted [4]. For the planning of harvesting of wild plants and the production of agricultural products, optimization models are of scientific and practical importance, allowing us to find the best options for the agricultural producer in the face of uncertainty. In a number of scientific articles at the theoretical and empirical levels, models for optimizing the production of products, including agricultural, with deterministic and indefinite parameters for making managerial decisions, are considered [5-9]. Moreover, in scientific work [5], it is proposed to convert some mathematical programming problems with interval parameters into deterministic problems. The authors in article [6] proposed parametric programming models for optimizing food production.

\footnotetext{
* Corresponding author: sofia.1987@bk.ru
} 
Meanwhile, many coefficients and the right parts of the mathematical programming model are uncertain, therefore, for an adequate assessment of the real situation, it is necessary to determine their statistical properties. Another problem is that the coefficients for unknowns in extreme problems when considering the planning of agricultural production [7] and harvesting of wild plants [8] are characterized by different variability. It is easy to assume a higher uncertainty of indicators characterizing the harvesting of wildgrowing food resources in comparison with data reflecting agricultural production. In works $[3,9]$ it was shown that when evaluating labor costs for harvesting wild plants, the yield of berries, mushrooms, pine nuts and medicinal plants, the areas of wild plants, expert opinion can be used. A similar method of determining indicators of agricultural production is less applicable.

It should be borne in mind that the harvesting of wild food resources has great prospects for export [4]. Therefore, for a significant development of the activities of the commodity producer, a promising direction is the combination of agricultural production and the receiving of wild plants in natural and processed form. Obviously, an additional function is of interest to an agricultural enterprise if it can consistently receive certain volumes of forest products. In this regard, the Irkutsk region has good prospects. Another question is how far agricultural producers are prepared for changes that do not require large risks. Guided by the document [4] in the Irkutsk region, certain conditions are created for enterprises to expand their functions in relation to obtaining food products.

In work [10], for the first time, a model was proposed for optimizing the combination of agricultural production and harvesting of wild food resources. This article is a continuation of research in this direction to improve models and expand their applications. Its goal is to build a model to optimize the combination of agricultural production and harvesting of wild food resources based on the properties of the variability of the coefficients with unknowns to increase the efficiency of managing agricultural enterprises.

To achieve the goal, the following tasks were solved: 1) building a model to optimize the receipt of food products in the face of uncertainty; 2) application of the model on the example of an agricultural enterprise in the Irkutsk region.

\section{Materials}

The article are used data on wild-growing food resources of municipal districts of the Irkutsk Region, prices for wild plants, labor costs for harvesting, distribution areas of taiga resources [2,3], as well as historical information on the receipt of production by animal industrial farms [1].. In addition to this, we single out the problem of the form of marketing of wild plants [11], including for export [12]. Meanwhile, by analogy with the solution of the problem of providing the local population with work [13], the same task is in a slightly different form for the Irkutsk region.

Although climate variability towards warming is not so significant in the region, especially in the north, nevertheless, it is necessary to study possible scenarios of spatiotemporal changes in species and yields of wild-growing products, as the authors of the article emphasize [14].

Obviously, to build models to optimize the combination of agricultural production and harvesting of wild plants, it is necessary to use mathematical programming methods in conditions of uncertainty. Here are some examples of modeling of receiving products with random and interval parameters [15-19]. In work [15], it was proposed to use stochastic methods in network planning of industrial production. The author of the article [16] pays great attention to multistage stochastic programming. For the convenience of solving environmental problems with uncertain parameters, special software is used described in work [17]. 
The applied model [18] shows the significant influence of meteorological factors, primarily precipitation, on land use strategies in Namibia, which is important for other countries and regions of the world. In some cases, one should also take into account the discreteness of some parameters in extreme problems [19].

In addition, literary sources were used to model the activities of an agricultural producer under conditions of uncertainty caused by many external factors [3, 6-9]. It is also worth highlighting the work [10], which presents a model for optimizing receiving of food products with interval estimates.

To assess the production and economic parameters of the activities of an agricultural producer, information from financial statements was used.

In the article the methods of probability theory and mathematical statistics, as well as methods of mathematical programming in conditions of uncertainty are applied.

\section{Results and Discussion}

A model is proposed, the objective function of which is the maximal income from agricultural production and harvesting of wild plants. As restrictions, land, fuel and wild resources were used. The conditions for labor resources and providing them with finances include data on the activities of procurers and producers. Inequality in the volume of production of various types of products for sale, including for export, is highlighted.

To increase the production of food products in the presence of large reserves of food forest resources, the producer must effectively combine the activities of agricultural production and harvesting of wild plants. We will build a model for optimizing the production of food products with an objective function in the form of income.

Since the indicators characterizing the variability of agricultural production and harvesting of wild food resources can be described using interval estimates [3, 8, 9], we present a model for optimizing the production of food products with interval parameters. In general terms, it can be written as follows. The objective function is focused on the maximum income:

$$
\sum_{s \in S} \sum_{l \in L} \widetilde{c}_{s l} x_{s l}+\sum_{n \in N} \sum_{l \in L} \widetilde{c}_{n l} x_{n l} \rightarrow \max
$$

where $\widetilde{c}_{s l}, \widetilde{c}_{n l}$ are specific income from agricultural production of type $s$ using technology $l$ and harvesting of wild-growing food resources of type $n$, varying within the lower and upper estimates $\widetilde{\widetilde{c}}_{s l} \leq \widetilde{c}_{s l} \leq \overline{\widetilde{c}}_{s l}$ и $\widetilde{\widetilde{c}}_{n l} \leq \widetilde{c}_{n l} \leq \overline{\widetilde{c}}_{n l}$.

The conditions for the limited nature of all agricultural and forest lands are as follows:

$$
\begin{aligned}
& \sum_{s \in S} \sum_{l \in L} \widetilde{v}_{s l} x_{s l}+\sum_{n \in N} \sum_{l \in L} \widetilde{r}_{n l} x_{n l} \leq B, \\
& \sum_{n \in N} \sum_{l \in L} \widetilde{r}_{n l} x_{n l} \leq A \quad(A \in B),
\end{aligned}
$$

where $\widetilde{v}_{s l}, \widetilde{r}_{n l}$ are logical coefficients characterizing the necessary area to obtain a unit of production by different technologies $l$, varying within the lower and upper values $\underline{\widetilde{v}}_{s l} \leq \widetilde{v}_{s l} \leq \overline{\widetilde{v}}_{s l}, \underline{\widetilde{r}}_{n l} \leq \widetilde{r}_{n l} \leq \overline{\widetilde{r}}_{n l}$.

The conditions of production of the final product of at least a given volume are: 
limited labor costs:

$$
\sum_{s \in S} \widetilde{c}_{s l} x_{s l}+\sum_{n \in N} \widetilde{c}_{n l} x_{n l} \geq \widetilde{V}_{l}(l \in L),
$$

$$
\sum_{s \in S} \widetilde{\alpha}_{s l} x_{s l}+\sum_{n \in N} \widetilde{\beta}_{n l} x_{n l} \leq D_{l} \quad(l \in L)
$$

by condition of material costs:

$$
\sum_{s \in S} \widetilde{u}_{s l} x_{s l}+\sum_{n \in N} \widetilde{b}_{n l} x_{n l} \leq W_{l} \quad(l \in L),
$$

where $\tilde{V}_{l}$ are production of agricultural and wild-growing products of at least a given volume in monetary terms, varying within $\underline{\widetilde{V}}_{l} \leq \widetilde{V}_{l} \leq \overline{\widetilde{V}}_{l} ; \tilde{\alpha}_{s l}$ are labor costs per unit of agricultural production, varying within $\underline{\alpha}_{s l} \leq \widetilde{\alpha}_{s l} \leq \overline{\widetilde{\alpha}}_{s l} ; \tilde{\beta}_{n l}$ are labor costs for the procurement of a unit of wild food products, varying within $\underline{\widetilde{\beta}}_{n l} \leq \widetilde{\beta}_{n l} \leq \overline{\widetilde{\beta}}_{n l} ; \tilde{u}_{s l}$ and $\tilde{b}_{n l}$ are material costs for the production of a unit of agricultural and wild products, varying within $\underline{\tilde{u}}_{s l} \leq \tilde{u}_{s l} \leq \overline{\widetilde{u}}_{s l}$ и $\underline{\tilde{b}}_{n l} \leq \widetilde{b}_{n l} \leq \overline{\widetilde{b}}_{n l}$.

The condition that the variables are non-negative is

$$
x_{s l}, x_{n l} \geq 0 \text {. }
$$

In the proposed model, the coefficients for unknowns in the objective function are generally variable values. It is shown that they can be described as interval indicators.

Meanwhile, the coefficients for unknowns in the constraints associated with the production of agricultural products are random variables [7]. In some cases, it is possible to describe the harvesting indicators of wild-growing food products using the laws of probability distribution [2]. Then, for the case with averaged coefficients for unknowns' objective function, model (1) - (7) will take the following form.

The optimality criterion is written as

$$
\sum_{s \in S} \sum_{l \in L} c_{s l} x_{s l}+\sum_{n \in N} \sum_{l \in L} c_{n l} x_{n l} \rightarrow \max
$$

where $c_{s l}, c_{n l}$ are average specific income from agricultural production and harvest of wild plants.

The restrictions will take the following form: for agricultural and forest land:

$$
\begin{gathered}
\sum_{s \in S} \sum_{l \in L} v_{s i l}^{p} x_{s l}+\sum_{n \in N} \sum_{l \in L} r_{n l}^{p} x_{n l} \leq B, \\
\sum_{n \in N} \sum_{l \in L} r_{n l}^{p} x_{n l} \leq A \quad(A \in B),
\end{gathered}
$$

where $v_{s l}^{p}, r_{n l}^{p}$ are logical coefficients characterizing the necessary area to obtain a unit of agricultural and forest products, $p$ is probability;

on the necessary volumes of production, labor costs and material costs:

$$
\sum_{s \in S} c_{s l} x_{s l}+\sum_{n \in N} c_{n l} x_{n l} \geq V_{l}(l \in L),
$$




$$
\begin{aligned}
& \sum_{s \in S} \alpha_{s l} x_{s l}+\sum_{n \in N} \beta_{n l} x_{n l} \leq D_{l} \quad(l \in L), \\
& \sum_{s \in S} u_{s l} x_{s l}+\sum_{n \in N} b_{n l} x_{n l} \leq W_{l} \quad(l \in L),
\end{aligned}
$$

where $V_{l}$ is production of agricultural and wild-growing products of at least a given volume in monetary terms, $\alpha_{s l}$ are average labor costs per unit of agricultural production, $\beta_{n l}$ are average labor costs for the procurement of a unit of wild food products, $u_{s l}$ and $b_{n l}$ are average material costs for the production of a unit of agricultural and wild products. The condition that the variables are non-negative is defined by inequality (7).

The mathematical model (7), (8) - (13) is a linear programming task with random variables. The problem is the stochastic estimation of the logical coefficients $r_{n l}^{p}$ that characterize the bio-productivity of wild plants. The solution to the problem can be based on the assumption that the series of productivity of food wild-growing resources are stationary and their compliance with the laws of probability distribution for the volumes of harvested forest products and bio-productivity. In work [20], for the description of perennial rows of harvesting of berries, mushrooms and pine nuts in the Irkutsk region according to the data given in the book [1], the probability distribution laws were proposed: normal, logarithmically normal, and gamma.

Let us turn to another model, which is a deterministic task of parametric programming due to the assumptions of low variation of coefficients with unknowns or their averaging and the possibility of describing some indicators using significant regression dependencies $[6]$.

We use the model (7) and (8) - (13), in which inequalities (9) and (10) are transformed to the form:

$$
\begin{aligned}
& \sum_{s \in S} \sum_{l \in L} v_{s l}(t) x_{s l}+\sum_{n \in N} \sum_{l \in L} r_{n l} x_{n l} \leq B, \\
& \sum_{n \in N} \sum_{l \in L} r_{n l} x_{n l} \leq A \quad(A \in B),
\end{aligned}
$$

where $t$ is parameter.

Model (7), (8), (11) - (13), (14), (15) involves the use of one parameter. At the same time, it is possible to apply deterministic multi-parameter linear programming problems to describe real situations.

Obviously, to solve the problems of optimizing agricultural production and harvesting wild food resources, parametric programming models with interval and probability coefficients for unknowns in constraints are applicable.

In particular, if inequality (14) is transformed to the form

$$
\sum_{s \in S} \sum_{l \in L} v_{s l}(t) x_{s l}+\sum_{n \in N} \sum_{l \in L} r_{n l}^{p} x_{n l} \leq B
$$

then (7), (8), (10) - (13), (16) is a model of parametric programming with probability coefficients for unknowns in the left-hand sides of constraints.

In the given models, the greatest variation is in the specific labor costs for harvesting wild resources and agricultural products, crop yields, bio productivity of wild plants and others. Thus, the optimization model for the production of agricultural and forest products 
is a linear programming problem with interval, random or averaged values. In this case, the option of using the parametric programming task is possible.

Each model has its own characteristics, and can be applied depending on the properties of the coefficients with unknown constraints.

For its implementation, methods for solving mathematical programming problems using the statistical test method were used.

The developed models were applied for CJSC "Irkutsk Semena". At the same time, their implementation used real data on agricultural production and potential indicators for harvesting wild food resources based on an assessment of the possible volumes of wild plants in the Irkutsk region [2], in which the farm operates. The following linear programming problems are solved under uncertainty:

1) the problem of parametric programming with interval estimates (1), (4)-(7), (14) and (15);

2) the problem of parametric programming with random coefficients for the unknowns in the left-hand sides of constraints (7), (8), (10)-(13), (16).

Tables 1 and 2 show the simulation results. Note that the intersectoral model that characterizes the production of crop products and the harvest of wild plants is considered. In the problem with interval estimates, the coefficients for the unknowns in left-hand sides of the constraints and the objective function were used as variable indicators: yield: potatoes, rape, perennial grasses for seeds, berries and mushrooms; labor costs for production (procurement) of products; remuneration for wild products; product prices. Crop yields were determined using a power-law trend expression $v_{s l}=a_{0} t^{a_{1}}$, in which $t$ is time, $a_{0}, a_{l}$ are the coefficients. Using this regression equation, which is significant on the basis of Fisher's criterion, a predictive value of the yield of agricultural crops used in the optimization model was obtained.

Table 1. The results of solving the problem of parametric programming with interval indicators in modeling the combination of agricultural production and harvesting of wild food resources at CJSC "Irkutsk semena"

\begin{tabular}{|c|c|c|c|c|c|c|c|c|}
\hline \multirow{3}{*}{ Estimate } & \multicolumn{7}{|c|}{ Plan, centner } & \multirow{3}{*}{$\begin{array}{l}\text { The value of } \\
\text { the objective } \\
\text { function, } \\
\text { million } \\
\text { rubles }\end{array}$} \\
\hline & Cereals & Potatoes & Rapeseed & \begin{tabular}{|c|} 
Perennia \\
1 herbs \\
on seeds
\end{tabular} & Berries & Mushrooms & $\begin{array}{c}\text { Medicinal } \\
\text { plants }\end{array}$ & \\
\hline & $x_{1}$ & $x_{2}$ & $x_{3}$ & $x_{4}$ & $x_{5}$ & $x_{6}$ & $x_{7}$ & \\
\hline Lower & 30750 & 61650 & 300 & 30 & 800 & 142 & 50 & 118.363 \\
\hline Upper & 33573 & 199476 & 3050 & 30 & 800 & 3201 & 50 & 395.396 \\
\hline Median & 30750 & 219088 & 1211 & 30 & 800 & 1279 & 50 & 345.992 \\
\hline
\end{tabular}

In order to obtain optimal solutions to the problem, multiple modeling is used with random determination of interval values by the Monte Carlo method. Some results from one hundred cases are shown in Table 1. The discrepancies between the lower and upper estimates of the objective function and the corresponding optimal plans are significant. Maximum farm outcomes depend on the level of production of cereals, potatoes and rapeseed. Wild plants have a great influence on production, especially on the volume of mushroom harvesting. The obtained solutions are interesting in that they allow us to identify optimal estimates of interval indicators. In this case, the decision-maker may choose a different option for obtaining food products, which, for example, may be between the median and the top mark.

A model with probabilistic quantities is less flexible, but the solutions obtained are related to a probability, which to some extent partially removes the uncertainty of the 
problem. Table 2 shows the simulation results for the parametric programming problem with random variables.

Yields of potatoes, berries and mushrooms are probabilistic values. They are modeled randomly based on the law of probability distribution. A normal distribution is proposed for potatoes and berries, and a logarithmically normal law is used for mushrooms. As in the model with interval indicators, the yield of grain crops was obtained by the power regression expression.

Some simulation results are given for the probability of joint events of $0.2,0.5$, and 0.8 . The solutions obtained for 700 cases using the statistical test method do not differ in the strong variability of the optimal plans. This can be explained by the fact that the decisions mainly depend on the yield of potatoes, berries and mushrooms, since the remaining indicators are deterministic. Although for the probabilities of joint events exceeding 0.95 , the objective function can reach values of more than 250 million rubles. In particular, at $p=0.984$, the optimality criterion is 264.378 million rubles. Never the less, the first results in this direction contribute to better planning, identifying different options for obtaining food products in favorable and unfavorable conditions.

Table 2. The results of solving the problem of parametric programming with probabilistic estimates when modeling the combination of agricultural production and harvesting of wild food resources in CJSC "Irkutsk semena". of the Irkutsk district

\begin{tabular}{|c|c|c|c|c|c|c|c|c|}
\hline \multirow{3}{*}{$\begin{array}{l}\text { Probabil } \\
\text { ity of } \\
\text { joint } \\
\text { events } p\end{array}$} & \multicolumn{7}{|c|}{ Plan, centner } & \multirow{3}{*}{$\begin{array}{l}\text { The value of the } \\
\text { objective } \\
\text { function, million } \\
\text { rubles }\end{array}$} \\
\hline & Cereals & Potatoes & Rapeseed & $\begin{array}{c}\text { Perennial } \\
\text { herbs on } \\
\text { seeds }\end{array}$ & Berries & Mushrooms & $\begin{array}{l}\text { Medicina } \\
1 \text { plants }\end{array}$ & \\
\hline & $x_{I}$ & $x_{2}$ & $x_{3}$ & $x_{4}$ & $x_{5}$ & $x_{6}$ & $x_{7}$ & \\
\hline \multicolumn{9}{|c|}{ Cereal yield is determined by the trend equation (equal to $20.5 \mathrm{c} / \mathrm{ha}$ ) } \\
\hline 0.20 & 16500 & 56750 & 300 & 30 & 150 & 76 & 40 & 88.039 \\
\hline 0.50 & 30750 & 67500 & 3050 & 50 & 150 & 150 & 243 & 138.173 \\
\hline 0.80 & 30750 & 67500 & 3050 & 50 & 150 & 2271 & 40 & 183.499 \\
\hline
\end{tabular}

The probabilistic model allows you to assess the risks of the agricultural enterprise

To improve the model of parametric programming with random values, it is necessary to take into account the variation in product prices, labor costs for production and harvesting. In this case, you can use the parametric programming problem with interval and probabilistic estimates. It is clear that such a task complicates the obtaining of solutions and requires a special interpretation of the results.

\section{Conclusion}

The possibility of agricultural producers on the production of agricultural products and the harvesting of wild food resources is considered.

An optimization model for the production of agricultural and forest products with interval indicators characterizing the coefficients for unknowns in the objective function and the left parts of the constraints is built. As interval estimates, specific incomes from obtaining a unit of production, labor costs for the production of agricultural products and harvesting of wild plants, and productivity of forest resources and crops were used.

An optimization model for combining the production of agricultural products and forest resources with probabilistic estimates, which were used as the bio-productivity of agricultural and wild food resources, was created. The conditions for the application of this model are determined. 
Parametric programming problems with interval and probabilistic estimates are proposed. As a parameter, time was used, and the predicted indicator was the yield of grain crops. These tasks are used to optimize the combination of agricultural and food wildgrowing products to CJSC "Irkutsk Semena".

Modeling using extreme problems with interval and probabilistic indicators allows us to determine the optimal solutions from the set. More flexible is the model with interval coefficients. The use of the probabilistic model is limited by the lack of long-term data, primarily on the yield of wild plants.

A promising direction is the development of models with interval and random estimates.

The developed models can be used as a mathematical support of information management system for obtaining agricultural products and wild plants.

\section{Acknowledgment}

The some results study was obtained with funded by RFBR according to the research project no. 19-07-00322

\section{References}

1. N.A. Kruzhkovand, Yu.E. Vashukevich, The history of the Irkutsk trust of the cooperfarming enterprises (2013)

2. Ya. M. Ivanyo, Regional models of clusters of procurement, processing and sale of wild food products (2019)

3. Ya. Ivanyo and, S.Petrova, Atlantis Press, 169, 108 (2019)

4. Decree of the Government of the Irkutsk region of October 26, 2018 N 772-pp On approval of the state program of the Irkutsk region "Development of agriculture and regulation of agricultural products, raw materials and food markets" for 2019 - 2024" (2019)

5. V.I Levin, Management, communication and security systems, 4, 123 (2015)

6. Ya. M. Ivanyo, M.N. Barsukova, Bulletin of ISTU, 4, 57 (2017)

7. V. R. Elokhin, Izvestiya Irkutsk State Economic Academy, 3, 137 (2009)

8. Ya. M. Ivanyo and, E.K. Boltvina, Bulletin of ISTU, 6(113), 73 (2016)

9. Ya. Ivanyo, N. Bendik, P. Asalkhanov, Management of the agro-industrial enterprise: optimization, uncertainty, expert assessments, 6 (2019)

10. M.N. Barsukova, E.N. Ivanova, S.A. Petrova, Actual problems of agricultural science, 33, 40 (2019)

11. D. L. Zubov, A. A. Maksaev, A.V. Tkach, Fundamental and applied research of the cooperative sector of the economy, 1, 3 (2019)

12. K.F.Wiersum, Human Ecology, 45(6), 787 (2017)

13. A.Nygren, C.Lacuna-Richman, K.Keinänen, L.Alsa, Small-scale Forest Economics, Management and Policy, 5 (2), 249 (2006)

14. Manish Das, Vanita Jain and S. K. Malhotra, Indian Journal of Agricultural Sciences, 86 (11), 1375 (2016)

15. A. I. Borodin and N.Nikolaevna, The Model of Stochastic Optimization of Production under Uncertainty and Risk Asian Social Science, 14(5), 33 (2018)

16. Nikolaos V. Sahinidis, Computers and Chemical Engineering, 28, 971 (2004) 
17. C.James, Hydrology Days, 17 (2015)

18. Stéphanie Domptailand Ernst-August Nuppenau, Ecological Economics, Elsevier, 69 (12), 2475 (2010)

19. N. Chiadamrongand V. Piyathanavong, Journal of Industrial Engineering International, 13, 465 (2017)

20. Ya. Ivanyo, Scientific and Practical Journal «Actual Issues of Agricultural Science», 25, 62 (2017) 